\title{
A comparison of endoanal ultrasound and computed tomography in staging rectal cancer and in clinical decision making - a preliminary study
}

D P Wickramasinghe ${ }^{1}$, D N Samarasekera ${ }^{1}$

(Index words: rectal cancer, CT imaging, endoanal ultrasound)

\begin{abstract}
Introduction The treatment options and the prognosis of rectal cancer $(R C)$ depend mainly on the stage. Computed tomography (CT) has been the main staging tool in RC but endoanal ultrasound (EAUS) is thought to be more accurate.

Methods Patients with histologically proven rectal cancer presenting for the first time were staged using CT and EAUS. TNM staging was used to stage the rectal cancer.

Results 24 patients (M:F 1:1) with a mean age of 57.3 (range $=23-80, S D=15.3$ ) years were included. The majority had a tumour of stage IIA/T3NOMO (CT = 10, EAUS = 12). The staging of the tumour was the same in both investigations in 11 patients, while in 8 patients, EAUS staging was higher. The agreement for the $\mathrm{T}$ and $\mathrm{N}$ stages were kappa 0.24 and 0.5 respectively. There was a moderate and fair agreement between the overall TNM staging (weighted kappa 0.435) and the treatment strategies (weighted kappa 0.226) respectively, based on each imaging method. Of the 13 patients whose staging was different, the management changed in 6 $(25 \%)$ patients $(p=0.016)$. CT identified distal metastases in 2 patients.
\end{abstract}

Conclusions EAUS and CT have only a fair to moderate agreement for staging and deciding treatment. However, EAUS has a significant influence when deciding treatment protocols.

Ceylon Medical Journal 2012; 57: 33-35

\section{Introduction}

Colorectal cancer is the third highest cause of cancer related deaths in the world. The incidence in Sri Lanka has risen from 0.7/100,000 in 1985 to 2.4/100,000 in 2005 [1]. Although computed tomography (CT) has been the main modality of rectal cancer staging since 1981, recently, endoanal ultrasound (EAUS) and magnetic resonance imaging (MRI) have made their presence felt in a major way [2-4]. In Sri Lanka, there are only 8 CT scanners available in the public health sector and only 2 centres with EAUS facilities. Therefore CT remains the most available and widely used imaging modality in rectal cancer staging.

Previous studies that compared CT imaging and EAUS in the staging of rectal cancer have failed to describe the impact of either imaging modality on patient management [5].

Therefore, the aims of our study were (i) to identify the correlation between stages and treatment strategy based on each imaging modality and (ii) to look at the impact of EAUS imaging on clinical decision making and patient management.

\section{Methods}

All patients with histologically proven rectal cancer presenting for the first time for EAUS imaging were included in the study. In addition, they underwent CT evaluation within 2 weeks of the date of EAUS assessment. The Ethics Review Committee of the hospital approved the study protocol. EAUS was performed by a single investigator using the 360 degree $10 \mathrm{MHz}$ Olympus GFUM 20 endoanal probe (Olympus America Inc., Pennsylvania). CT imaging was done using a 16 slice CT scanner (Activion, Toshiba). Each interpreter was blinded to the findings of the other investigator.

Inter-rater reliability analysis using the kappa statistic was performed to determine consistency among the patient's tumour and nodal stages and the TNM stage as determined by CT and EAUS. There is a general consensus on stage oriented protocols for adjuvant and neo-adjuvant chemo-radiotherapy and depending on the staging of each imaging technique, all the patients were categorised into 3 treatment groups; (i) surgery alone (ii) surgery with adjuvant chemo-radiotherapy (ACRT) or (iii) palliative treatment $[3,5]$. The two treatment modalities as determined by CT staging were then compared with treatment modality determined by EAUS using weighted kappa. Fisher's exact test was used to assess the association between CT and EAUS staging and the change in the management plan.

${ }^{1}$ University Surgical Unit, The National Hospital of Sri Lanka, Colombo.

Correspondence: DNS, e-mail: <samarasekera58@yahoo.co.uk>. Received 4 August and revised version accepted 9 December 2011. Competing interests: none declared. 
Statistical analyses were performed using MedCalc for Windows, version 11.3.0.0 (MedCalc Software, Mariakerke, Belgium).

\section{Results}

Twenty four patients (M:F 1:1) were included in the study. The average age was 57.3 (range 23-80, SD- 15.3) years. The majority had a tumour of stage II-A/ T3N0M0 (10 CT staging and 12 EAUS staging) (Table). The staging of the tumour was the same in both imaging modalities in 11 patients and in 8 patients, EAUS staging of the tumour was higher than the CT staging.
The inter-rater reliability for the T stage was found to be kappa $=0.24$ (95\% CI-0.0675 to 0.559) and for the $\mathrm{N}$ stage was kappa $=0.5$ (95\% CI 0.162-0.838). There was a moderate agreement between the overall TNM staging between CT and EAUS (weighted kappa 0.435, 95\% CI 0.135 to 0.735 ) and a fair agreement between the treatment strategies based on each imaging method (weighted kappa $0.226,95 \%$ CI -0.137 to 0.588 ).

Of the 13 patients whose stagings were different, the management was changed in 6 (25\% of the total study sample, $\mathrm{p}=0.016$ ). When there was a change in the $\mathrm{T}$ stage, the management changed in 5 patients and this was also statistically significant $(p=0.015)$. However, when the $\mathrm{N}$ stage changed, the management changed only in 1 patient $(p=1.0)$.

Table. Summary of patients, their staging and change in management

\begin{tabular}{|c|c|c|c|c|}
\hline Patient number & CT staging & EAUS staging & Outcome & Change in management \\
\hline 1 & T4 N0 M0 & T3 N0 Mx & EAUS stage lower & No \\
\hline 2 & T3 N0 M0 & T3 M0 Nx & Stages equal & No \\
\hline 3 & T3 N0 M0 & T3 N0 Mx & Stages equal & No \\
\hline 4 & T3 N0 M0 & T3 N0Mx & Stages equal & No \\
\hline 5 & T2 N0 M0 & T3 N1 Mx & EAUS stage higher & Yes \\
\hline 6 & T4 N0 M0 & T4 N1 Mx & EAUS stage higher & No \\
\hline 7 & T3 N0 M0 & T4 N1 Mx & EAUS stage higher & No \\
\hline 8 & T3 N0Mx & T3 N0 Mx & Stages equal & No \\
\hline 9 & T2 N0 Mx & T3 N0 Mx & EAUS Stage higher & Yes \\
\hline 10 & T3 N0 Mx & T3 N1 Mx & EAUS stage higher & No \\
\hline 11 & T3 N0 Mx & T3 N0 Mx & Stages equal & No \\
\hline 12 & T3N1 Mx & T3 N1Mx & Stages equal & No \\
\hline 13 & T4 N0 M0 & T3 N0 Mx & EAUS stage lower & No \\
\hline 14 & T3 N1 Mx & T3 N1 Mx & Stages equal & No \\
\hline 15 & T4 N1 M1 & T3 N1 Mx & EAUS stage lower & Yes \\
\hline 16 & T3 N0Mx & T3 N0 Mx & Stages equal & No \\
\hline 17 & T2 N0 Mx & T2 N0 Mx & Stages equal & No \\
\hline 18 & T0 N0 M0 & T3 N0 Mx & EAUS stage higher & Yes \\
\hline 19 & T4 N0 Mx & T3 N0 Mx & EAUS stage lower & No \\
\hline 20 & T4 N0Mx & T4 N0 Mx & Stages equal & No \\
\hline 21 & T3 N0Mx & T3 N0 Mx & Stages equal & No \\
\hline 22 & T3 N0Mx & T3 N1 Mx & EAUS stage higher & No \\
\hline 23 & T3 N1 M1 & T3N1Mx & EAUS stage lower & Yes \\
\hline 24 & T2 N0 Mx & T4 N0 Mx & EAUS stage higher & Yes \\
\hline
\end{tabular}




\section{Discussion}

The stage of the rectal cancer is the single preoperative predictor of survival after therapy [2]. A recent meta-analysis reported sensitvity and specificity of MRI to be equal to EAUS in detecting adjacent organ and lymph node involvement and that EAUS is superior to CT (and even MRI) in assessing local invasion [4].

There is consensus that the $\mathrm{T}$ staging is the most important factor determining treatment and, the lack of agreement raises concerns about the optimal staging, especially for resource poor centres/countries which only have facilities for CT [6]. The management protocol changed significantly $(p=0.015)$ when the $T$ stage was different and there were equal numbers of patient $(n=3)$ in each group who had either a more invasive or a less invasive therapeutic option.

Of the 9 patients in whom the EAUS detected positive lymph nodes, CT had only identified 4 patients. Other authors report better nodal staging with EAUS [7]. Of the 13 patients whom had different staging in each method, in 4, the management changed to a more costly/risky/invasive strategy. This is slightly lower than the value described (63.3\%) [8]. The use of preoperative chemo-radiotherapy is a well identified factor affecting staging accuracy [9]. We did not include patients who presented with recurrences following surgery and/or radiotherapy because it was neither financially nor logistically possible to repeat either of the imaging following neoadjuvant therapy.

\section{Conclusion}

There was a fair agreement between EAUS and CT for $\mathrm{T}$ staging but a moderate agreement was shown for the $\mathrm{N}$ stage, a moderate agreement between the overall TNM staging and a fair agreement between the treatment strategies based on each imaging modality. There was a significant change in the management protocol which was due to EAUS staging. However, distal metastases are still best detected by CT scans. Therefore, prior to planning the management of a patient with carcinoma of the rectum both EAUS and CT may be considered complementary to make an accurate assessment of local and distal spread respectively.

\section{References}

1. Cancer Incidence Data: Sri Lanka Year 2001-2005, Colombo: Cancer Registry - National Cancer Control Programme.

2. Stewart DY, Yan M, Mutch I, et al. Predictors of diseasefree survival in rectal cancer patients undergoing curative proctectomy. Colorectal Disease 2008; 10: 879-86.

3. Tjandra JJ, Kilkenny JW, Buie WD, et al. Practice parameters for the management of rectal cancer (revised). Diseases of Colon and Rectum 2005; 48: 411-23.

4. Bipat S, Glas AS, Slors FJ, et al. Rectal cancer: local staging and assessment of lymph node involvement with endoluminal US, CT, and MR imaging - a meta-analysis. Radiology 2004; 232: 773-83.

5. Kapiteijn ECA, Marijnen ID, Nagtegaal H, et al. Preoperative radiotherapy combined with total mesorectal excision for resectable rectal cancer. New England Journal of Medicine 2001; 345: 638-46.

6. Rovera FG, Dionigi L, Boni S, et al. The role of EUS and MRI in rectal cancer staging. Surgical Oncology 2007; 16 (Suppl) S51-2.

7. Pappalardo GD, Reggio FM, Frattaroli A, et al. The value of endoluminal ultrasonography and computed tomography in the staging of rectal cancer: a preliminary study. Journal of Surgical Oncology 1990; 43: 219-22.

8. Shami VM, Parmar KS, Waxman I. Clinical impact of endoscopic ultrasound and endoscopic ultrasound-guided fine-needle aspiration in the management of rectal carcinoma. Diseases of the Colon and Rectum 2004; 47: 965.

9. Napoleon B, Pujol B, Berger F, et al. Accuracy of endosonography in the staging of rectal cancer treated by radiotherapy. British Journal of Surgery 1991; 78: 785-8. 